\title{
Random Fixed Point Theorem for Weakly Compatible Mappings under Implicit Relation in Cone Random Metric Spaces
}

\author{
R. A. Rashwan ${ }^{1, *}$, H. A. Hammad ${ }^{2}$ \\ ${ }^{1}$ Department of Mathematics, Faculty of Science, Assuit University, Assuit 71516, Egypt \\ ${ }^{2}$ Department of Mathematics, Faculty of Science, Sohag University, Sohag 82524, Egypt
}

Copyright (C)2017 by authors, all rights reserved. Authors agree that this article remains permanently open access under the terms of the Creative Commons Attribution License 4.0 International License

\begin{abstract}
In this paper, we establish a unique common random fixed point theorem in c one $r$ andom m etric spaces for four weakly compatible mappings by using an implicit relation. Some corollaries of this theorem for two and three random weakly compatible mappings are obtained. Some examples are given to support our generalization. Our results presented in this paper extend and improve several recent results in the setting of cone random metric spaces.
\end{abstract}

Keywords Random Fixed Point, Cone Random Metric Spaces, Random Weakly Compatible Mappings, Implicit Relation

\section{Introduction}

Random nonlinear analysis is an important mathematical discipline which is mainly concerned with the study of random nonlinear operators and their properties and is much needed for the study of various classes of random equations. Developments in the investigation on fixed points of non-expansive mappings, contractive mappings in different spaces like metric spaces, Banach spaces, Fuzzy metric spaces and cone metric spaces have almost been saturated. The study of random fixed point theorems was initiated by the Prague school of probabilistic in 1950's [9, 10, 31]. The introduction of randomness leads to several new questions of measurability of solutions, probabilistic and statistical aspects of random solutions. Common random fixed point theorems are stochastic generalization of classical common fixed point theorems. Random methods have revolutionized the financial markets. The survey article by Bharucha-Reid [8] in 1976 attracted the attention of several mathematicians and gave wings to the theory. The results of Špaček and Hanš in multi-valued contractive mappings was extended by
Itoh [14]. Now this theory has become the full fledged research area and various ideas associated with random fixed point theory are used to give the solution of nonlinear system see [5-7, 11, 25] and nonlinear integral equation see [19-21]. Common random fixed points and random coincidence points of a pair of compatible random operators and fixed point theorems for contractive random operators in Polish spaces are obtained by Papageorgiou $[17,18]$ and Beg $[3,4]$.

Huang and Zhang [12] improved the concept of metric spaces by replacing the set of real numbers with an ordered Banach space, hence they have defined the cone metric spaces. They also described the convergence of sequences and introduced the notion of completeness in cone metric spaces. They have proved some fixed point theorems of contractive mappings on complete cone metric space with the assumption of normality of a cone. According to this concept, several other authors $[1,13,24,30]$ studied the existence of fixed points and common fixed points of mappings satisfying contractive type condition on a normal cone metric space. In 2008 , the assumption of normality in cone normal spaces is deleted by Rezapour and Hamlbarani [24], which is an important event in developing fixed point theory in cone metric spaces.

Random fixed point results in cone random metric spaces are stochastic generalization of deterministic fixed point results in cone metric space. Several other authors see [15, 22, 26-29] studied the existence of random fixed points and common random fixed points of mappings satisfying contractive type conditions in setting cone random metric spaces.

In continuation of these results, we extend the contraction condition via implicit relation by Saluja and Tripathi [28] to four random operators and obtain a common random fixed point for four weakly compatible mappings on a nonempty separable closed subset of cone random metric spaces. 


\section{Preliminaries}

\subsection{Definition [16]}

Let $(E, \tau)$ be a topological vector space. A subset $p$ of $E$ is called a cone if the following conditions satisfied:

(c $\left.c_{1}\right) p$ is closed, nonempty and $p \neq\{0\}$;

( $\left.\mathrm{c}_{2}\right) a, b \in \mathbb{R}, a, b \geq 0$ and $x, y \in p \Rightarrow a x+b y \in p$;

$\left(\mathrm{c}_{3}\right)$ If $x \in p$ and $-x \in p \Rightarrow x=0$.

For a given cone $p \subset E$, we define a partial ordering $\leq$ with respect to $p$ by $x \leq y$ iff $y-x \in p$. We shall write $x<y$ to indicate that $x \leq y$ but $x \neq y$, while $x \ll y$ will stand for $y-x \in p^{\circ}$, where $p^{\circ}$ indicate to the interior of $p$.

\subsection{Definition $[12,32]$}

Let $X$ be a nonempty set and the mapping the mapping $d: X \times X \rightarrow E$ satisfies:

$\left(\mathrm{d}_{1}\right) 0 \leq d(x, y)$ for all $x, y \in X$ and $d(x, y)=0 \Leftrightarrow$ $x=y$;

$\left(\mathrm{d}_{2}\right) d(x, y)=d(y, x)$ for all $x, y \in X$;

$\left(\mathrm{d}_{3}\right) d(x, y) \leq d(x, z)+d(z, y) x, y, z \in X$.

Then $d$ is called a cone metric [12] or $K$-metric [32] on $X$ and $(X, d)$ is called a cone metric space [12].

The concept of a cone metric space is more general than that of a metric space, because each metric space is a cone metric space where $E=\mathbb{R}$ and $p=[0,+\infty)$.

\subsection{Example [12]}

Let $E=\mathbb{R}^{2}, p=\left\{(x, y) \in \mathbb{R}^{2}: x \geq 0, y \geq 0\right\}$, $X=\mathbb{R}$ and $d: X \times X \rightarrow E$ defined by $d(x, y)=$ $(|x-y|, \mu(|x-y|))$ where $\mu \geq 0$ is a constant. Then $(X, d)$ is a cone metric space with normal cone $p$ where $K=1$.

\subsection{Example [23]}

Let $E=l^{2}, p=\left\{\left\{x_{n}\right\}_{n \geq 1} \in E^{2}: x_{n} \geq 0\right.$, for all $\mathrm{n}\},(X, \rho)$ a metric space and $d: X \times X \rightarrow E$ defined by $d(x, y)=\left\{\frac{\rho(x, y)}{2^{n}}\right\}_{n \geq 1}$. Then $(X, d)$ is a cone metric space.

Clearly, the above examples present that the class of cone metric spaces contains the class of metric spaces.

\subsection{Definition [12]}

Let $(X, d)$ be a cone metric space. We say that $\left\{x_{n}\right\}$ is:

(i) a Cauchy sequence if for every $\varepsilon$ in $E$ with $0 \ll \varepsilon$, then there is an $\mathbb{N}$ such that for all $n, m>\mathbb{N}, d\left(x_{n}, x_{m}\right) \ll \varepsilon$;

(ii) a convergent sequence if for every $\varepsilon$ in $E$ with $0 \ll \varepsilon$, then there is an $\mathbb{N}$ such that for all $n>\mathbb{N}, d\left(x_{n}, x\right) \ll \varepsilon$ for some fixed $x$ in $X$.

A cone metric space $X$ is said to be complete if every Cauchy sequence in $X$ is convergent in $X$.

The following definitions are given in [16].

\subsection{Definition (Measurable function)}

Let $(\Omega, \Sigma)$ be a measurable space with $\Sigma$-a sigma algebra of subsets of $\Omega$ and $M$ be a nonempty subset of a metric space $X=(X, d)$. Let $2^{M}$ be the family of nonempty subsets of $M$ and $C(M)$ the family of all nonempty closed subsets of $M$. A mapping $G: \Omega \rightarrow 2^{M}$ is called measurable if for each open subset $U$ of $M, G^{-1}(U) \in \Sigma$, where $G^{-1}(U)=\{\omega \in$ $\Omega: G(\omega) \cap U \neq \varnothing\}$.

\subsection{Definition (Measurable selector)}

A mapping $\xi: \Omega \rightarrow M$ is called measurable selector of a measurable mappings $G: \Omega \rightarrow 2^{M}$ if $\xi$ is measurable and $\xi(\omega) \in G(\omega)$ for each $\omega \in \Omega$.

\subsection{Definition (Random operator)}

The mapping $T: \Omega \times M \rightarrow X$ is called a random operator iff for each fixed $x \in M$, the mapping $T(., x): \Omega \rightarrow X$ is measurable.

\subsection{Definition (Continuous random mapping)}

A random operator $T: \Omega \times M \rightarrow X$ is called continuous random operator if for each fixed $x \in M$ and $\omega \in \Omega$, the mapping $T(\omega,):. \Omega \rightarrow X$ is continuous.

\subsection{Definition (Random fixed point)}

A measurable mappings $\xi: \Omega \rightarrow M$ is a random fixed point of a random operator $T: \Omega \times M \rightarrow X$ iff $T(\omega, \xi(\omega))=$ $\xi(\omega)$ for each $\omega \in \Omega$.

\subsection{Definition (Cone random metric space)}

Let $M$ be a nonempty set and the mapping $d: \Omega \times M \rightarrow p$, where $p$ is a cone, $\omega \in \Omega$ be a selector, satisfy the following conditions:

(i) $d(x(\omega), y(\omega)) \geq 0$ and $d(x(\omega), y(\omega))=0 \Leftrightarrow x(\omega)=$ $y(\omega)$ for all $x(\omega), y(\omega) \in \Omega \times M$,

(ii) $d(x(\omega), y(\omega))=d(y(\omega), x(\omega))$ for all $x, y \in M$, $\omega \in \Omega$ and $x(\omega), y(\omega) \in \Omega \times M$, (iii) $d(x(\omega), y(\omega)) \leq$ $d(x(\omega), z(\omega))+d(z(\omega), y(\omega))$ for all $x, y, z \in M$ and $\omega \in \bar{\Omega}$ be a selector,

(iv) for any $x, y \in M, \omega \in \Omega, d(x(\omega), y(\omega))$ is nonincreasing and left continuous.

Then $d$ is called cone random metric on $M$ and $(M, d)$ is called a cone random metric space.

\subsection{Definition (Weakly compatible [2])}

Random operators $T, S: \Omega \times X \rightarrow X$ are weakly compatible if $T(S(\xi(\omega)))=S(T(\xi(\omega)))$ provided that $T(\xi(\omega))=$ $S(\xi(\omega))$ for every $\omega \in \Omega$. 


\subsection{Definition (Implicit relation)}

We say an implicit relation between $x$ and $y$ is given for all $x, y \geq 0$, if there exists a real number $0<\lambda<1$ such that $x \leq \lambda y$, whenevere $\phi:\left(\mathbb{R}^{+}\right)^{4} \rightarrow \mathbb{R}^{+}$be a family of real valued continuous functions, nondecreasing in the first argument and satisfy the following axioms:

$$
\begin{gathered}
x \leq \phi\left(y, \frac{x+y}{2}, 0, x+y\right) \text { or } x \leq \phi\left(y, \frac{x+y}{2}, y, x\right) \\
\text { or } x \leq \phi\left(y, \frac{x}{2}, y, x\right) \text { or } x \leq \phi(x, y, x, x) .
\end{gathered}
$$

\section{Main Results}

In this section we shall prove a common random fixed point theorem under implicit relation for four weakly compatible mappings satisfying some conditions in the setting of cone random metric spaces.

\subsection{Theorem}

Let $(X, d)$ be a complete cone random metric space with respect to a cone $p$ and let $M$ be a nonempty separable closed subset of $X$. Assume that $S, T, P$ and $Q$ be four continuous random operators defined on $M$ such that for $\omega \in \Omega$, $S(\omega,),. T(\omega,),. P(\omega,),. Q(\omega,):. \Omega \times M \rightarrow M$ satisfying the following conditions:

(i) $S(\omega, X) \subseteq Q(\omega, X)$ and $T(\omega, X) \subseteq P(\omega, X)$,

(ii) the pairs $\{S, P\}$ and $\{T, Q\}$ are random weakly compatible mappings,

(iii)

$$
d\left(S(x(\omega), T(y(\omega))) \leq \phi\left(\begin{array}{c}
d(P(x(\omega)), Q(y(\omega))), \frac{1}{2}\{d(P(x(\omega)), S(x(\omega)))+d(Q(y(\omega)), T(y(\omega)))\} \\
d(P(x(\omega)), T(y(\omega))), d(Q(y(\omega)), S(x(\omega)))
\end{array}\right),\right.
$$

for all $x(\omega), y(\omega) \in \Omega \times X$. Then the four random mappings have a unique common random fixed point in $X$.

Proof. For each $x_{\circ}(\omega), x_{1}(\omega) \in \Omega \times X$ and $n=0,1,2, .$. we choose $y_{1}(\omega), y_{2}(\omega) \in \Omega \times X$ such that $y_{1}(\omega)=$ $S\left(x_{\circ}(\omega)\right)=Q\left(x_{1}(\omega)\right)$ and $y_{2}(\omega)=T\left(x_{1}(\omega)\right)=P\left(x_{2}(\omega)\right)$. In general we construct a sequence of measurable mappings $y_{n}(\omega), x_{n}(\omega): \Omega \rightarrow X$ defined by

$$
\left\{\begin{array}{c}
y_{2 n+1}(\omega)=S\left(x_{2 n}(\omega)\right)=Q\left(x_{2 n+1}(\omega)\right) \\
y_{2 n+2}(\omega)=T\left(x_{2 n+1}(\omega)\right)=P\left(x_{2 n+2}(\omega)\right)
\end{array},\right.
$$

Then from (3.1) and (3.2), we get

$$
\begin{aligned}
d\left(y_{2 n+1}(\omega), y_{2 n+2}(\omega)\right) & =d\left(S\left(x_{2 n}(\omega)\right), T\left(x_{2 n+1}(\omega)\right)\right) \\
& \leq \phi\left(\begin{array}{c}
1 \\
\frac{1}{2}\left\{d\left(P\left(x_{2 n}(\omega)\right), S\left(x_{2 n}(\omega)\right)\right)+d\left(Q\left(x_{2 n+1}(\omega)\right), T\left(x_{2 n+1}(\omega)\right)\right)\right\}, \\
d\left(P\left(x_{2 n}(\omega)\right), T\left(x_{2 n+1}(\omega)\right)\right), d\left(Q\left(x_{2 n+1}(\omega)\right), S\left(x_{2 n}(\omega)\right)\right)
\end{array}\right) \\
& =\phi\left(\begin{array}{c}
d\left(y_{2 n}(\omega), y_{2 n+1}(\omega)\right), \frac{1}{2}\left\{d\left(y_{2 n}(\omega), y_{2 n+1}(\omega)\right)+d\left(y_{2 n+1}(\omega), y_{2 n+2}(\omega)\right)\right\}, \\
d\left(y_{2 n}(\omega), y_{2 n+2}(\omega)\right), d\left(y_{2 n+1}(\omega), y_{2 n+1}(\omega)\right)
\end{array}\right) \\
& \leq \phi\left(\begin{array}{r}
d\left(y_{2 n}(\omega), y_{2 n+1}(\omega)\right), \frac{1}{2}\left\{d\left(y_{2 n}(\omega), y_{2 n+1}(\omega)\right)+d\left(y_{2 n+1}(\omega), y_{2 n+2}(\omega)\right)\right\}, 0, \\
d\left(y_{2 n}(\omega), y_{2 n+1}(\omega)\right)+d\left(y_{2 n+1}(\omega), y_{2 n+2}(\omega)\right)
\end{array}\right) .
\end{aligned}
$$

So, from Definition 2.13, we can write

$$
d\left(y_{2 n+1}(\omega), y_{2 n+2}(\omega)\right) \leq \lambda d\left(y_{2 n}(\omega), y_{2 n+1}(\omega)\right) .
$$

Similarly, we have

$$
d\left(y_{2 n}(\omega), y_{2 n+1}(\omega)\right) \leq \lambda d\left(y_{2 n-1}(\omega), y_{2 n}(\omega)\right)
$$

hence

$$
d\left(y_{2 n+1}(\omega), y_{2 n+2}(\omega)\right) \leq \lambda^{2} d\left(y_{2 n-1}(\omega), y_{2 n}(\omega)\right) .
$$

On continuing this process, we have

$$
d\left(y_{2 n+1}(\omega), y_{2 n+2}(\omega)\right) \leq \lambda^{2 n} d\left(y_{0}(\omega), y_{1}(\omega)\right) .
$$


Also, for $n>m$, we get

$$
\begin{aligned}
d\left(y_{n}(\omega), y_{m}(\omega)\right) & \leq d\left(y_{n}(\omega), y_{n-1}(\omega)\right)+d\left(y_{n-1}(\omega), y_{n-2}(\omega)\right)+\ldots+d\left(y_{m+1}(\omega), y_{m}(\omega)\right) \\
& \leq\left(\lambda^{n-1}+\lambda^{n-2}+\ldots+\lambda^{m}\right) d\left(y_{1}(\omega), y_{\circ}(\omega)\right) \\
& \leq\left(\frac{\lambda^{m}}{1-\lambda}\right) d\left(y_{1}(\omega), y_{\circ}(\omega)\right) .
\end{aligned}
$$

Let $0 \ll \varepsilon$ is given. Choose a natural number $N$ such that $\left(\frac{\lambda^{m}}{1-\lambda}\right) d\left(y_{1}(\omega), y_{\circ}(\omega)\right) \ll \varepsilon$ for every $m \geq N$, hence

$$
d\left(y_{n}(\omega), y_{m}(\omega)\right) \leq\left(\frac{\lambda^{m}}{1-\lambda}\right) d\left(y_{1}(\omega), y_{\circ}(\omega)\right) \ll \varepsilon,
$$

this implies that $\left\{y_{n}(\omega)\right\}$ is a Cauchy sequence in $\Omega \times X$.

Since $(X, d)$ is complete, then there exists $z(\omega) \in \Omega \times X$ such that $y_{n}(\omega) \rightarrow z(\omega)$ as $n \rightarrow \infty$. Then from (3.2), we get

$$
\begin{aligned}
\lim _{n \rightarrow \infty} S\left(x_{2 n}(\omega)\right) & =\lim _{n \rightarrow \infty} Q\left(x_{2 n+1}(\omega)\right)=z(\omega) \\
\text { and } \lim _{n \rightarrow \infty} T\left(x_{2 n+1}(\omega)\right) & =\lim _{n \rightarrow \infty} P\left(x_{2 n+2}(\omega)\right)=z(\omega),
\end{aligned}
$$

therefore

$$
\lim _{n \rightarrow \infty} S\left(x_{2 n}(\omega)\right)=\lim _{n \rightarrow \infty} Q\left(x_{2 n+1}(\omega)\right)=\lim _{n \rightarrow \infty} T\left(x_{2 n+1}(\omega)\right)=\lim _{n \rightarrow \infty} P\left(x_{2 n+2}(\omega)\right)=z(\omega) .
$$

Since $T(\omega, X) \subseteq P(\omega, X)$, then there exists $u(\omega) \in \Omega \times X$ such that

$$
z(\omega)=P(u(\omega)) .
$$

From (3.1), we obtain

$$
\begin{aligned}
& d(S(u(\omega)), z(\omega)) \\
\leq & d\left(S(u(\omega)), T\left(x_{2 n+1}(\omega)\right)\right)+d\left(T\left(x_{2 n+1}(\omega)\right), z(\omega)\right) \\
\leq & \phi\left(\begin{array}{c}
1 \\
\left.\frac{1}{2}\left\{d\left(P(u(u)), Q\left(x_{2 n+1}(\omega)\right)\right), S(u(\omega))\right)+d\left(Q\left(x_{2 n+1}(\omega)\right), T\left(x_{2 n+1}(\omega)\right)\right)\right\}, \\
d\left(P(u(\omega)), T\left(x_{2 n+1}(\omega)\right)\right), d\left(Q\left(x_{2 n+1}(\omega)\right), S(u(\omega))\right)
\end{array}\right)+d\left(T\left(x_{2 n+1}(\omega)\right), z(\omega)\right) .
\end{aligned}
$$

Taking the limit as $n \rightarrow \infty$ in above inequality and using (3.3) and (3.4), we get

$$
d(z(\omega), S(u(\omega))) \leq \phi\left(0, \frac{d(z(\omega), S(u(\omega)))}{2}, 0, d(z(\omega), S(u(\omega)))\right) \leq 0 .
$$

Thus $-d(z(\omega), S(u(\omega))) \in p$. But $d(z(\omega), S(u(\omega))) \in p$, therefore by Definition $2.1\left(\mathrm{c}_{3}\right)$, we have $d(z(\omega), S(u(\omega)))=0$ and so $z(\omega)=S(u(\omega))$.

From (3.4) we have

$$
z(\omega)=P(u(\omega))=S(u(\omega)) .
$$

Hence $u(\omega)$ is a random coincidence point of $P$ and $S$.

Since the pair $(P, S)$ is random weakly compatible, i.e. $P(S(u(\omega)))=S(P(u(\omega)))$ this gives

$$
P(z(\omega))=S(z(\omega)) .
$$

Again since $S(\omega, X) \subseteq Q(\omega, X)$, then there exists $v(\omega) \in \Omega \times X$ such that

$$
z(\omega)=Q(v(\omega))
$$

From (3.1), (3.5) and (3.7), we have

$$
\begin{aligned}
d(z(\omega), T(v(\omega))) & =d(S(u(\omega)), T(v(\omega))) \\
\leq & \phi\left(\begin{array}{c}
\frac{1}{2}\{d(P(u(\omega)), S(u(\omega))), Q(v(\omega))), \\
d(P(u(\omega)), T(v(\omega))), d(Q(v(\omega)), T(v(\omega)))\}, S(u(\omega)))
\end{array}\right) \\
& =\phi\left(0, \frac{d(z(\omega), T(v(\omega)))}{2}, d(z(\omega), T(v(\omega))), 0\right) \leq 0 .
\end{aligned}
$$


this implies that $d(z(\omega), T(v(\omega))) \leq 0$, thus $-d(z(\omega), T(v(\omega))) \in p$. But $d(z(\omega), T(v(\omega))) \in p$, therefore by Definition 2.1 $\left(\mathrm{c}_{3}\right)$, we have $d(z(\omega), T(v(\omega)))=0$ and so $z(\omega)=T(v(\omega))$.

From (3.7) we get

$$
z(\omega)=Q(v(\omega))=T(v(\omega)) .
$$

Hence $v(\omega)$ is a random coincidence point of $T$ and $Q$.

Since the pair $T$ and $Q$ are random weakly compatible, i.e. $T(Q(v(\omega)))=Q(T(v(\omega)))$ this implies that

$$
T(z(\omega))=Q(z(\omega)) .
$$

Now we show that $z(\omega)$ is a random fixed point of $S$, we have from (3.1) that

$$
\begin{aligned}
d(S(z(\omega)), z(\omega)) & =d(S(z(\omega)), T(v(\omega))) \\
\leq & \phi\left(\begin{array}{c}
d(P(z(\omega)), Q(v(\omega))), \\
\frac{1}{2}\{d(P(z(\omega)), S(z(\omega)))+d(Q(v(\omega)), T(v(\omega)))\}, \\
d(P(z(\omega)), T(v(\omega))), d(Q(v(\omega)), S(z(\omega)))
\end{array}\right) .
\end{aligned}
$$

Using (3.6) and (3.8), we get

$$
d(S(z(\omega)), z(\omega)) \leq \phi(d(S(z(\omega)), z(\omega)), 0, d(S(z(\omega)), z(\omega)), d(z(\omega), S(z(\omega)))) \leq 0,
$$

it follows that $d(S(z(\omega)), z(\omega))=0$, i.e. $S(z(\omega))=z(\omega)$.

According to (3.6), we obtain that

$$
P(z(\omega))=S(z(\omega))=z(\omega) .
$$

By a similar way and using (3.10), we can prove that for all $\omega \in \Omega$,

$$
T(z(\omega))=Q(z(\omega))=z(\omega) .
$$

The equations (3.10) and (3.11) shows that $z(\omega)$ is a common random fixed point of $T, S, P$ and $Q$.

For uniqueness. Let $q(\omega) \neq z(\omega)$ be another common random fixed point of the four mappings, then from (3.1), one can write

$$
\begin{aligned}
& d(z(\omega), q(\omega))=d(S(z(\omega)), T(q(\omega))) \\
& \leq \phi\left(\begin{array}{c}
d(P(z(\omega)), Q(q(\omega))), \frac{1}{2}\{d(P(z(\omega)), S(z(\omega)))+d(Q(q(\omega)), T(q(\omega)))\}, \\
d(P(z(\omega)), T(q(\omega))), d(Q(q(\omega)), S(z(\omega)))
\end{array}\right) \\
& =(d(z(\omega), q(\omega)), 0, d(z(\omega), q(\omega)), d(q(\omega), z(\omega))) \leq 0,
\end{aligned}
$$

a contradiction. Hence $q(\omega)=z(\omega)$ and so $z(\omega)$ is a unique common random fixed point of $T, S, P$ and $Q$. The proof is completed.

If we take, $P=Q$ in above theorem we obtain the following corollary.

\subsection{Corollary}

Let $(X, d)$ be a complete cone random metric space with respect to a cone $p$ and let $M$ be a nonempty separable closed subset of $X$. Assume that $S, T$ and $P$ are three continuous random operators defined on $M$ such that for $\omega \in \Omega$, $S(\omega,),. T(\omega,),. P(\omega,):. \Omega \times M \rightarrow M$ satisfying the following conditions:

(i) $S(\omega, X) \subseteq P(\omega, X)$ and $T(\omega, X) \subseteq P(\omega, X)$,

(ii) the pairs $\{S, P\}$ and $\{T, P\}$ are random weakly compatible mappings,

(iii)

$$
d(S(x(\omega)), T(y(\omega))) \leq \phi\left(\begin{array}{c}
d(P(x(\omega)), P(y(\omega))) \\
\frac{1}{2}\{d(P(x(\omega)), S(x(\omega)))+d(P(y(\omega)), T(y(\omega)))\} \\
d(P(x(\omega)), T(y(\omega))), d(P(y(\omega)), S(x(\omega)))
\end{array}\right)
$$

for all $x(\omega), y(\omega) \in \Omega \times X$. Then the three random mappings have a unique common random fixed point in $X$. Putting $P=Q$ and $S=T$ in above theorem we get the following corollary. 


\subsection{Corollary}

Let $(X, d)$ be a complete cone random metric space with respect to a cone $C$ and let $M$ be a nonempty separable closed subset of $X$. Assume that $S$ and $P$ are two continuous random operators defined on $M$ such that for $\omega \in \Omega, S(\omega,),. P(\omega,$.$) :$ $\Omega \times M \rightarrow M$ satisfying the following conditions:

(i) $S(\omega, X) \subseteq P(\omega, X)$,

(ii) the pair $\{S, P\}$ is random weakly compatible mappings,

(iii)

$$
d(S(x(\omega)), S(y(\omega))) \leq \phi\left(\begin{array}{c}
d(P(x(\omega)), P(y(\omega))), \frac{1}{2}\{d(P(x(\omega)), S(x(\omega)))+d(P(y(\omega)), S(y(\omega)))\} \\
d(P(x(\omega)), S(y(\omega))), d(P(y(\omega)), S(x(\omega)))
\end{array}\right)
$$

for all $x(\omega), y(\omega) \in \Omega \times X$. Then the two random mappings have a unique common random fixed point in $X$.

\subsection{Remark}

Letting $P=Q=I$ (where $I$ is the identity mapping defined by $I(\omega, x)=x(\omega)$ for all $\omega \in \Omega$ ) and eliminating the conditions (i), (ii) in our theorem, we obtain the results of Saluja and Tripathi [28].

Finally, we present some examples to verify the requirements of our theorem as follows.

\subsection{Example}

Let $(\Omega, \Sigma)$ denotes a measurable space and $M=\{1,2,3,4,5\} \subset R$ with the usual metric $d$. Consider $\Omega=\{1,2,3,4,5\}$ and let $\sum$ be the sigma algebra of Lebesgue's measurable subset of $\Omega$. Define $T, Q, F, P: \Omega \times M \rightarrow M$ for all $\omega \in \Omega$ by

$$
\begin{aligned}
& S(\omega, x)=\left\{\begin{array}{l}
3 \text { if } x=1 \\
4 \text { otherwise }
\end{array} \text { and } Q(\omega, x)=\left\{\begin{array}{l}
5 \text { if } x=1 \\
4 \text { otherwise }
\end{array}\right.\right. \\
& T(\omega, x)=\left\{\begin{array}{l}
1 \text { if } x=1 \\
4 \text { otherwise }
\end{array} \text { and } P(\omega, x)=\left\{\begin{array}{l}
2 \text { if } x=1 \\
4 \text { otherwise }
\end{array}\right.\right.
\end{aligned}
$$

Taking measurable sequence $x_{n}(\omega)=x(\omega)=3$, it's clearly that $S(\omega, x) \subseteq Q(\omega, x)$ and $T(\omega, x) \subseteq P(\omega, x)$ and for all $\omega \in \Omega, S\left(x_{n}(\omega)\right)=P\left(x_{n}(\omega)\right)=4, S\left(P\left(x_{n}(\omega)\right)\right)=P\left(S\left(x_{n}(\omega)\right)\right)=4$, this implies that $P$ and $S$ are random weakly compatible mappings, similarly $T$ and $Q$ too. To satisfy the condition (1.3), by taking $x(\omega)=1$ and $y(\omega)=M-\{1\}$, we can write

$$
\begin{aligned}
1 & =d\left(S(x(\omega), T(y(\omega))) \leq \phi\left(\begin{array}{c}
d(P(x(\omega)), Q(y(\omega))), \frac{1}{2}\{d(P(x(\omega)), S(x(\omega)))+d(Q(y(\omega)), T(y(\omega)))\} \\
d(P(x(\omega)), T(y(\omega))), d(Q(y(\omega)), S(x(\omega)))
\end{array}\right)\right. \\
& =\phi\left(2, \frac{1}{2}, 2,1\right) .
\end{aligned}
$$

From Definition 2.13, we can write $1 \leq 2 \lambda$, hence $\lambda=\frac{1}{2} \in(0,1)$, therefore all conditions of Theorem 3.1 are satisfied and 4 is a unique random fixed point of $S, T, P$ and $Q$.

\subsection{Example}

Let $(\Omega, \Sigma)$ be a measurable space and $M=\{2,3,4,5,7\} \subset R$ with the usual metric $d$. Consider $\Omega=\{2,3,4,5,7\}$ and let $\sum$ be the sigma algebra of Lebesgue's measurable subset of $\Omega$. Define $T, Q, S, P: \Omega \times M \rightarrow M$ for all $\omega \in \Omega$, by

$$
\begin{aligned}
& S(\omega, x)=\left\{\begin{array}{l}
2 \text { if } x=2 \\
4 \text { otherwise }
\end{array} \text { and } Q(\omega, x)=\left\{\begin{array}{l}
5 \text { if } x=2 \\
4 \text { otherwise }
\end{array}\right.\right. \\
& T(\omega, x)=\left\{\begin{array}{l}
3 \text { if } x=2 \\
4 \text { otherwise }
\end{array} \text { and } P(\omega, x)=\left\{\begin{array}{l}
7 \text { if } x=2 \\
4 \text { otherwise }
\end{array}\right.\right.
\end{aligned}
$$

By taking measurable sequence $x_{n}(\omega)=x(\omega)=1$, then it's obvious that $S(\omega, x) \subseteq Q(\omega, x), T(\omega, x) \subseteq P(\omega, x)$ and for all $\omega \in \Omega, S\left(x_{n}(\omega)\right)=P\left(x_{n}(\omega)\right)=4, S\left(P\left(x_{n}(\omega)\right)\right)=P\left(S\left(x_{n}(\omega)\right)\right)=4$, this implies that $P$ and $S$ are random weakly 
compatible mappings, similarly $T$ and $Q$ too. To justify the condition (1.3), by taking $x(\omega)=2$ and $y(\omega)=M-\{2\}$, it follows that

$$
\begin{aligned}
2 & =d(S(x(\omega)), T(y(\omega))) \leq \phi\left(\begin{array}{c}
d(P(x(\omega)), Q(y(\omega))), \\
\frac{1}{2}\{d(P(x(\omega)), S(x(\omega)))+d(Q(y(\omega)), T(y(\omega)))\}, \\
d(P(x(\omega)), T(y(\omega))), d(Q(y(\omega)), S(x(\omega)))
\end{array}\right) \\
& =\phi\left(3, \frac{5}{2}, 3,2\right)=\phi\left(3, \frac{2+3}{2}, 3,2\right) .
\end{aligned}
$$

By Definition 2.13, we get $2 \leq 3 \lambda$, hence $\lambda=\frac{2}{3} \in(0,1)$, therefore all requirements of Theorem 3.1 are satisfied and 4 is a unique random fixed point of $S, T, P$ and $Q$.

\subsection{Example}

Let $(\Omega, \Sigma)$ be a measurable space and $M=\Omega=[0,1] \subset R$ with the usual metric $d$, and let $\sum$ be the sigma algebra of Lebesgue's measurable subset of $\Omega$. Define $T, Q, S, P: \Omega \times M \rightarrow M$ for all $\omega \in \Omega$, by

$$
\begin{aligned}
& S(\omega, x)=\left\{\begin{array}{l}
\frac{2}{3} x(\omega) \text { if } x(\omega) \in \Omega-\{\omega\} \\
x(\omega) \text { if } x(\omega)=\omega
\end{array} \quad \text { and } Q(\omega, x)=\left\{\begin{array}{l}
1 \text { if } x(\omega) \in \Omega-\{\omega\} \\
x(\omega) \text { if } x(\omega)=\omega
\end{array}\right.\right. \\
& T(\omega, x)=\left\{\begin{array}{l}
0 \text { if } x(\omega) \in \Omega-\{\omega\} \\
x(\omega) \text { if } x(\omega)=\omega
\end{array} \text { and } P(\omega, x)=\left\{\begin{array}{l}
\frac{1}{3} x(\omega) \text { if } x(\omega) \in \Omega-\{\omega\} \\
x(\omega) \text { if } x(\omega)=\omega
\end{array}\right.\right.
\end{aligned}
$$

Let $x(\omega)=\omega$ be a measurable mapping, then it's obvious that $S(\omega, x) \subseteq Q(\omega, x), T(\omega, x) \subseteq P(\omega, x)$ and for all $\omega \in \Omega$, $S(x(\omega))=P(x(\omega))=\omega, S(P(x(\omega)))=P(S(x(\omega)))=\omega$, this implies that $P$ and $S$ are random weakly compatible mappings, similarly $T$ and $Q$ too. To justify the condition (1.3), by taking $x(\omega) \in \Omega-\{\omega\}$ and $y(\omega)=\omega$, it follows that

$$
\begin{aligned}
\frac{x(\omega)}{3} & =d(S(x(\omega)), T(y(\omega))) \leq \phi\left(\begin{array}{c}
d(P(x(\omega)), Q(y(\omega))), \\
\frac{1}{2}\{d(P(x(\omega)), S(x(\omega)))+d(Q(y(\omega)), T(y(\omega)))\}, \\
d(P(x(\omega)), T(y(\omega))), d(Q(y(\omega)), S(x(\omega)))
\end{array}\right) \\
& =\phi\left(\frac{2 x(\omega)}{3}, \frac{\frac{1}{3} x(\omega)}{2}, \frac{2 x(\omega)}{3}, \frac{x(\omega)}{3}\right) .
\end{aligned}
$$

Using Definition 2.13, we obtain $\frac{1}{3} x(\omega) \leq \frac{2}{3} x(\omega) \lambda$, hence $\lambda=\frac{1}{2} \in(0,1)$, therefore all conditions of Theorem 3.1 are satisfied and $\omega$ is a unique random fixed point of $S, T, P$ and $Q$. 


\section{Acknowledgements}

The authors would like to thanks the anonymous referees for their efforts and valuable suggestions to improve the quality of the paper.

\section{REFERENCES}

[1] M. Abbas, G. Jungck. Common fixed point results for non commuting mappings without continuity in cone metric spaces, J. Math. Anal. Appl., Vol.341, 416-420, 2008.

[2] M. A. Al-Thagafi, N. Shahzad. Generalized I-nonexpansive maps and invariant approximation, Acta Math. Sinica, Vol.25, No.5, 867-876, 2008.

[3] I. Beg. Random fixed points of random operators satisfying semicontractivity conditions, Math. Japonica, Vil.46, 151$155,1997$.

[4] I. Beg. Approximation of random fixed points in normed spaces, Nonlinear Anal., Vol.51, 1363-1372. 2002.

[5] I. Beg, M. Abbas. Equivalence and stability of random fixed point iterative procedures, J. Appl. Math. Stoch. Anal., Vol.2006, 1-19, 2006.

[6] I. Beg, M. Abbas. Iterative procedures for solutions of random operator equations in Banach spaces, J. Math. Anal. Appl., Vol.315, 181-201, 2006.

[7] A. T. Bharucha-Reid. Random Integral equations, Mathematics in Science and Engineering, Vol. 96, Academic Press, New York, 1972.

[8] A. T. Bharucha-Reid. Fixed point theorems in probabilistic analysis, Bull. Amer. Math. Soc., Vol.82, No.5, 641-657, 1976.

[9] O. Hanš. Reduzierende zufăllige transformationen, Czechoslov. Math. J., Vol.7, No.82, 154-158, 1957.

[10] O. Hanš. Random operator equations, Proceeding of the 4th Berkeiey Symposium on Mathematical Statistics and Probability, Vol.II, University of California Press, California, 185-202, 1961.

[11] C. J. Himmelberg. Measurable relations, Fundamenta Mathematicae, Vol.87, 53-72, 1975

[12] L. G. Huang, X. Zhang. Cone metric spaces and fixed point theorems of contractive mappings, J. Math. Anal. Appl., Vol.332, 1468-1476, 2007.
[13] D. Ilic, V. Rakocevic. Common fixed points for maps on cone metric space, J. Math. Anal. Appl., Vol.341, 876-882, 2008.

[14] S. Itoh. Random fixed point theorems with an application to random differential equations in Banach spaces, J. Math. Anal. Appl., Vol.67, No.2, 261-273, 1979.

[15] S. Mehta, A. D. Singh. Some fixed point theorems in cone random metric spaces, South Asian J. Math., Vol.2, No.3, 248-254, 2012.

[16] S. Mehta, A. D. Singh, Vanita Ben Dhagat. Fixed point theorems for weak contraction in cone random metric spaces, Bull. Math. Soc., Vol.103, No.4, 303-310, 2011.

[17] N. S. Papageorgiou. Random fixed point theorems for measurable multifunctions in Banach spaces, Proc. Amer. Math. Soc., Vol.97, 507-514, 1986.

[18] N. S. Papageorgiou, On measurable multifunctions with stochastic domain, J. Australian Math. Soc., Vol.45, 204-216, 1988 .

[19] R. A. Rashwan, D. M. Albaqeri. A common random ?xed point theorem and application to random integral equations, Int. J. Appl. Math. Reser., Vol.3, No.1, 71-80, 2014.

[20] R. A. Rashwan, H. A. Hammad. Random fixed point theorems with an application to a random nonlinear integral equation, Journal of Linear and Topological Algebra, Vol.5, No.2, 119133, 2016.

[21] R. A. Rashwan, H. A. Hammad. Random common fixed point theorem for random weakly subsequentially continuous generalized contractions with application, Int. J. Pure Appl. Math., Vol.109, No.4, 813-826, 2016.

[22] R. A. Rashwan, H. A. Hammad. A common random fixed point theorem for weakly compatible mappings in cone random metric spaces, Universal Journal of Computational Mathematics, Vol.4, No.4, 67-74, 2016.

[23] Sh. Rezapour. A review on topological properties of cone metric spaces, in Procceedingd of the International Conference on Analysis, Topology and Appl. (ATA 08), Vrinjacka Banja, Serbia, May-june 2008.

[24] Sh. Rezapour, R. Hamlbarani. Some notes on the paper, One metric spaces and fixed point theorems of contractive mappings, J. Math. Anal. Appl., Vol.345, 719-724, 2008.

[25] V. M. Sehgal, S. P. Singh. On random approximations and a random fixed point theorem for set valued mappings, Proc. Amer. Math. Soc., Vol.95, 91-94, 1985. 
[26] G. S. Saluja. Random fixed point theorems for Ciric quasi contraction in cone random metric spaces, AAnalele Universităţii de Vast, Timişoara Seria Matematică-Informatică, Vol.LIII, No.(1), 163-175, 2015.

[27] G. S. Saluja, On common random fixed point theorems under contractive type condition in cone random metric spaces, Gulf J. Math., Vol.3, No.(4), 111-122, 2015.

[28] G. S. Saluja, M. P. Tripathi, On common random fixed point results in cone random metric spaces, J. Fixed Point Theory, Vol.2014:4, 1-11, 2014.

[29] G. S. Saluja, M. P. Tripathi, Some common random fixed point theorems for contractive type conditions in cone random metric spaces, Acta. Univ. Sapientiae Math., Vol.8, No.1, 174-190, 2016.

[30] P. Vetro, Common fixed points in cone metric spaces, Rend. Circ. Mat. Palermo, Vol.56, 464-468, 2007.

[31] D. H. Wagner, Survey of measurable selection theorems, SIAM J. Control Optim., Vol.15, 859-903, 1977.

[32] P. P. Zabrejko, K-metric and K-normed linear spaces: survey, Collectanea Math., Vol.48, 825-859, 1997. 